\title{
Breathlessness measurement should be standardised for the level of exertion
}

\author{
To the Editor:
}

BeAumont et al. [1] and Schultz et al. [2] should be commended for evaluating the impact of inspiratory muscle training on clinically relevant outcomes in patients with chronic obstructive pulmonary disease (COPD). Chronic breathlessness [3] is a cardinal symptom in people with cardiopulmonary disease and an essential end-point for trials and clinical care. As discussed in one of the papers [1], there were limitations in that breathlessness was not measured at a standardised level of exertion. This limitation is commonly encountered in clinical trials and warrants wider attention.

The frequency, intensity and distress of breathlessness can be modified by the patient by adapting the level of physical activity and exertion. Worsening breathlessness often leads to a vicious circle of limited physical activity and increasing deconditioning with successive worsening of symptoms. On the other end, improved breathlessness (for example due to medical intervention) may enable the patient to be more active and to achieve a higher work rate before symptom threshold is reached. Cardiopulmonary exercise testing (CPET) has shown that despite having very different ventilatory and exercise capacities, people with or without disease terminate exercise at similar levels of maximal breathlessness [4]. Thus, the maximal symptom level measured at the end of the test has limited value in quantifying the severity of breathlessness or the response to therapy. The importance of measuring breathlessness at a standardised exertion was stressed in a recent Cochrane review, where oxygen alleviated breathlessness during exercise testing when measured at iso-time but not when measured at the end of the exercise test [5].

Breathlessness is often measured using tests without a standardised exercise protocol such as the 6-min walking test $(6 \mathrm{MWT})[5,6]$. The $6 \mathrm{MWT}$ was developed and validated for measuring exercise capacity [6], but its use for measuring breathlessness is problematic for the following reasons: 1) the 6MWT is self-paced and the actual work performed can vary markedly [6]; and 2) breathlessness is measured at the end of test. As the 6MWT elicits a physiological response similar to maximal exertion during an incremental bicycle ergometer test [7], breathlessness measured at end of a 6MWT is relatively unresponsive to change [6].

Importantly, the issue of non-standardised exertion applies also to questionnaires (uni- and multidimensional) of breathlessness during daily life. A decrease in measured breathlessness may reflect decreased activity or avoidance of situations that provoke symptoms rather than symptomatic improvement. Analogously, a lack of change in breathlessness (despite treatment) may reflect an actual symptom improvement, as the patient may be able to achieve higher levels of exertion before reaching the same level of breathlessness. This may, at least partly, explain the discordance between some treatments such as oxygen, where the effect on breathlessness is seen at standardised exertion (such as iso-time during cycle exercise test) but not when the symptom is measured using a 6MWT or a questionnaire in daily life [5]. Changes in provoking factors (for example the level of activity) could be measured using other instruments and accounted for indirectly. However, it is unknown how this could be performed reliably and the approach is likely to be less effective than direct standardisation. Failure to account for the level of exertion may cause less precise and spurious results, including false negative findings where potential beneficial treatments of chronic breathlessness may be overlooked.

The gold standard for measuring exertional breathlessness is CPET, which is unfeasible for routine use in clinical practice and large clinical trials. Emerging field tests for measuring exertional breathlessness [8],

@ERSpublications

Measurement of breathlessness should be standardised for level of exertion to obtain unbiased results and to evaluate the effect of medical interventions for symptom relief http://ow.ly/bBB730j36qh

Cite this article as: Ekström M, Elmberg V, Lindow $\mathrm{T}$, et al. Breathlessness measurement should be standardised for the level of exertion. Eur Respir J 2018; 51: 1800486 [https://doi.org/10.1183/ 13993003.00486-2018]. 
including a 3-min step test and 3-min constant-rate shuttle walk test, have shown responsiveness to changes in breathlessness from bronchodilation in COPD $[9,10]$. Validated multidimensional questionnaires such as the Multidimensional Dyspnea Profile could be used during standardised exertion. Measuring breathlessness at standardised exertion is of fundamental importance for assessing symptom severity, selecting and characterising patients in clinical studies, and optimising the clinical evaluation and care of patients with chronic breathlessness.

Magnus Ekström ${ }^{1}$, Viktor Elmberg ${ }^{2}$, Thomas Lindow ${ }^{3}$ and Per Wollmer ${ }^{4}$

${ }^{1}$ Dept of Respiratory Medicine and Allergology, Institution for Clinical Sciences, Lund University, Lund, Sweden. ${ }^{2}$ Dept of Clinical Physiology, Blekinge Hospital, Karlskrona, Sweden. ${ }^{3}$ Dept of Clinical Physiology, Institution for Clinical Sciences, Lund University, Lund, Sweden. ${ }^{4}$ Clinical Physiology and Nuclear Medicine Unit, Dept of Translational Medicine, Lund University, Lund, Sweden.

Correspondence: Magnus Ekström, Dept of Clinical Sciences, Division of Respiratory Medicine and Allergology, Lund University, Skane University Hospital, Lund 22100, Sweden. E-mail pmekstrom@gmail.com

Received: March 092018 | Accepted: March 092018

Conflict of interest: None declared.

\section{References}

1 Beaumont M, Mialon P, Le Ber C, et al. Effects of inspiratory muscle training on dyspnoea in severe COPD patients during pulmonary rehabilitation: controlled randomised trial. Eur Respir J 2018; 51: 1701107.

2 Schultz K, Jelusic D, Wittmann $\mathrm{M}$, et al. Inspiratory muscle training does not improve clinical outcomes in 3-week COPD rehabilitation: results from a randomised controlled trial. Eur Respir J 2018; 51: 1702000.

3 Johnson MJ, Yorke J, Hansen-Flaschen J, et al. Towards an expert consensus to delineate a clinical syndrome of chronic breathlessness. Eur Respir J 2017; 49: 1602277.

4 Faisal A, Alghamdi BJ, Ciavaglia CE, et al. Common mechanisms of dyspnea in chronic interstitial and obstructive lung disorders. Am J Respir Crit Care Med 2015; 193: 299-309.

5 Ekström M, Ahmadi Z, Bornefalk-Hermansson A, et al. Oxygen for breathlessness in patients with chronic obstructive pulmonary disease who do not qualify for home oxygen therapy. Cochrane Database Syst Rev 2016; 11: CD006429.

6 Puente-Maestu L, Palange P, Casaburi R, et al. Use of exercise testing in the evaluation of interventional efficacy: an official ERS statement. Eur Respir J 2016; 47: 429-460.

7 Holland AE, Spruit MA, Troosters T, et al. An official European Respiratory Society/American Thoracic Society technical standard: field walking tests in chronic respiratory disease. Eur Respir J 2014; 44: 1428-1446.

8 Perrault H, Baril J, Henophy S, et al. Paced-walk and step tests to assess exertional dyspnea in COPD. COPD 2009; 6: 330-339.

9 Borel B, Wilkinson-Maitland CA, Hamilton A, et al. Three-minute constant rate step test for detecting exertional dyspnea relief after bronchodilation in COPD. Int J Chron Obstruct Pulmon Dis 2016; 11: 2991-3000.

10 Sava F, Perrault H, Brouillard C, et al. Detecting improvements in dyspnea in COPD using a three-minute constant rate shuttle walking protocol. COPD 2012; 9: 395-400.

Copyright @ERS 2018

From the authors:

We would like to thank M. Ekström and colleagues for their correspondence with comments on our article entitled "Effects of inspiratory muscle training on dyspnoea in severe COPD patients during pulmonary rehabilitation: a controlled randomised trial" [1]. We agree with most of the points underlined by M. Ekström and colleagues.

As mentioned, a limitation of our study is that dyspnoea at exertion was not evaluated at the same level of effort. Indeed, we measured dyspnoea at exertion at the end of the 6-min walk test (6MWT), which does not allow comparison of dyspnoea at the same level of effort. The 6MWT is a well-standardised exercise test according to the guidelines by Holland et al. [2]. We performed the 6MWT as it is

\section{@ERSpublications}

Dyspnoea should be measured at a standardised level of exertion http://ow.ly/kpMQ30jWXau

Cite this article as: Beaumont M, Mialon P, Couturaud F. Breathlessness measurement should be standardised for the level of exertion. Eur Respir J 2018; 51: 1800820 [https://doi.org/10.1183/ 13993003.00820-2018]. 
recommended by the American Thoracic Society/European Respiratory Society task force, including standardised instructions which limit performance variations. Moreover, as the 6MWT is a submaximal test, consequently, dyspnoea measured at the end of the 6MWT is also submaximal. Thus, in our study, dyspnoea evaluated with multidimensional dyspnoea profile (MDP) questionnaire (sensory components) (data not published) was significantly improved in a similar extent at the end of the 6MWT in the two randomised groups despite an increase in the 6-min walk distance, without significant difference between the two groups. The best test to compare dyspnoea at the same level of exertion would be the cycloergometer endurance test (constant work rate exercise test), because we can measure dyspnoea at isotime and isowork between the beginning and the end of the pulmonary rehabilitation programme [3]. The endurance shuttle walk test allows this measure too, but less data are available.

M. Ekström and colleagues added that "The issue of non-standardised exertion applies also to questionnaires (uni- and multidimensional) of breathlessness during daily life." It is right but there are dyspnoea scales such as the modified Medical Research Council dyspnoea scale (despite the lack of sensibility of this scale) or London Chest of Activity of Daily Living (LCADL) scale which allow measurement of the impact of dyspnoea on activity of daily of life [4]. Information provided by the LCADL scale could be more informative for the clinician than dyspnoea itself.

About the field test mentioned by M. Ekström and colleagues for measuring exertional dyspnoea, we agree with their comments: the 3-min constant rate step test and 3-min constant rate shuttle walk test have shown responsiveness to changes in dyspnoea from bronchodilation, and are able to measure dyspnoea at the same level of exertion before and after a treatment; however, studies must be realised to show responsiveness to change in dyspnoea after pulmonary rehabilitation.

Lastly, we fully agree with the idea of using MDP questionnaire during standardised exertion to measure dyspnoea. A multicentre study will begin to determine minimal clinical important difference (MCID) for the MDP questionnaire. The LCADL questionnaire would also be used for measuring the impact of dyspnoea during activity of daily living.

Marc Beaumont $^{1}$, Philippe Mialon ${ }^{2}$ and Francis Couturaud ${ }^{3}$

${ }^{1}$ Pulmonary Rehabilitation unit, Morlaix Hospital Centre, European University of Occidental Brittany, Morlaix, France. ${ }^{2}$ Pulmonary Physiology unit, EA 2438, European University of Occidental Brittany, University Brest Centre, Brest, France. ${ }^{3}$ Dept of Internal Medicine and Chest Diseases, EA3878 (G.E.T.B.O.), CIC INSERM 0502, University Hospital of Brest, European University of Occidental Brittany, Brest, France.

Correspondence: Marc Beaumont, Morlaix Hospital Centre, Pulmonary Rehabilitation Unit, Kersaint Gilly, B.P. 97237, Morlaix 29672, France. E-mail: marc.beaumont@univ-brest.fr

Received: May 012018 | Accepted: May 012018

Conflict of interest: None declared.

\section{References}

1 Beaumont M, Mialon P, Le Ber C, et al. Effects of inspiratory muscle training on dyspnoea in severe COPD patients during pulmonary rehabilitation: controlled randomised trial. Eur Respir J 2018; 51: 1701107.

2 Holland AE, Spruit MA, Troosters T, et al. An official European Respiratory Society/American Thoracic Society technical standard: field walking tests in chronic respiratory disease. Eur Respir J 2014; 44: 1428-1446.

3 Puente-Maestu L, Palange P, Casaburi R, et al. Use of exercise testing in the evaluation of interventional efficacy: an official ERS statement. Eur Respir J 2016; 47: 429-460.

4 Beaumont M, Couturaud F, Jego F, et al. Validation of the french version of the London Chest Activity of Daily Living (LCADL) scale and of the Dyspnea-12 questionnaire. Int J Chron Obstruct Pulm Dis 2018; 13: 1399-1405. 